\title{
Tsafon
}

Revue d'études juives du Nord

$75 \mid 2018$

Exil des langues - Langues d'exil

\section{Ombres et lumières de la judaïcité belge dans la Grande Guerre}

Daniel Dratwa

\section{(2) OpenEdition \\ 12 Journals}

Édition électronique

URL : https://journals.openedition.org/tsafon/612

DOI : $10.4000 /$ tsafon. 612

ISSN : 2609-6420

Éditeur

Association Jean-Marie Delmaire

Édition imprimée

Date de publication : 1 juin 2018

Pagination : 113-128

ISSN : 1149-6630

\section{Référence électronique}

Daniel Dratwa, « Ombres et lumières de la judaïcité belge dans la Grande Guerre », Tsafon [En ligne],

75 | 2018, mis en ligne le 06 mai 2019, consulté le 30 juin 2021. URL : http://journals.openedition.org/ tsafon/612 ; DOI : https://doi.org/10.4000/tsafon.612

Tsafon. Revues d'études juives du Nord 


\author{
Varia : Histoire
}

\title{
Ombres et lumières de la judaïcité belge dans la Grande Guerre
}

\section{Daniel Dratwa*}

En hommage au baron Jean Bloch 'zl

Contrairement à ce qui s'est produit dans d'autres pays européens, l'historiographie tant belge qu'étrangère ne semble guère s'être intéressée à la période de 1914-1918 et à la collectivité juive de Belgique dans ce contexte. Même si, dans l'immédiat après-guerre, des plaques commémoratives ${ }^{1}$ des soldats tués au front sont apposées sur les murs de certaines synagogues et qu'une liste des militaires est dressée, pour contrer l'antisémitisme dans la seconde moitié des années 1930, la Shoah viendra oblitérer le travail mémoriel relatif à ce premier conflit du $\mathrm{XX}^{\mathrm{e}}$ siècle. Dans aucune des expositions que nous avons visitées ces derniers mois ni dans les catalogues consultés, nul ne parle de ce sujet ${ }^{2}$. Cent ans plus tard, il parait donc juste et utile de combler en partie cette lacune.

\footnotetext{
* Conservateur-fondateur du Musée Juif de Belgique (Bruxelles).

Sans l'invitation du professeur Marc Saperstein à participer au colloque international Rabbis and the Great War qui s'est tenu à Londres le 7 juillet 2014, je n'aurais sans doute pas mis par écrit un sujet qui me passionne depuis trente ans; voir à ce propos Daniel Dratwa, «Albert $\mathrm{I}^{\mathrm{er}}$ ou l'amour de la patrie », dans Regards $\mathrm{n}^{\circ} 11,23$ février 1984 , p. 11. Je remercie l'Ivor Foundation de m'avoir permis d'y participer. Une version anglaise sensiblement différente a été publiée dans European Judaism, vol. 48, number 1, spring 2015, p. 100-109.

${ }^{1}$ Voir la séance du Consistoire Central israélite de Belgique (CCIB) du 10 novembre 1920 dans le Registre des procès-verbaux n 8, Archives CCIB (A-CCIB).

${ }^{2}$ Depuis que j'ai rédigé cette contribution pour la conférence de Londres, ont été présentés au public le 18 novembre 2014 les articles de Jean-Philippe Schreiber et
} 
Quand le 4 août 1914, la Belgique est envahie, malgré la neutralité soutenue par les grandes puissances - dont l'Allemagne, depuis son indépendance en 1830 - le roi Albert $\mathrm{I}^{\text {er }}$ et les ministres appellent la France et l'Angleterre à la rescousse pour garantir cette neutralité. Il prononce alors au Parlement cette phrase devenue célèbre : «J'ai foi dans nos destinées. Un pays qui se défend s'impose au respect de tous. Ce pays ne périra pas. Dieu sera avec nous dans cette juste cause. Vive la Belgique indépendante ». La veille, face à l'ultimatum allemand, la population s'en prend aux résidents allemands, pourtant bien intégrés. Des vitrines sont brisées et quelques maisons sont saccagées. Partout, ou presque, une vague d'espionite aiguë s'empare des esprits.

Dès le 20 août, Bruxelles est occupée par les armées belligérantes et, le 10 octobre, la ville d'Anvers tombe aux mains de l'ennemi après que l'armée belge s'est retirée. Il faudra l'acte héroïque de deux Belges, provoquant une inondation, pour que le front se stabilise le 3 novembre sur l'Yser et que les ports de Nieuport et Dixmude ne passent pas sous contrôle de l'ennemi. Grâce à l'aide des Britanniques, une partie infime du territoire est sauvegardée - 38 communes $^{3}$ sur 2636 : le roi et son État-major installés à La Panne et les membres de son gouvernement repliés au Havre y organisent la résistance jusqu'en 1918. Selon l'historien Krzysztof Pomian : «La Belgique est donc le seul pays du conflit mondial à avoir été occupé dans sa quasi-totalité pendant plus de quatre ans » et ce fut une occupation implacable ${ }^{4}$.

La communauté juive de Belgique avant ces événements se compose d'environ 50.000 âmes, une population immigrée en majorité durant les trente dernières années ${ }^{5}$ :

\begin{tabular}{|c|c|c|c|}
\hline 1880 & 1900 & 1910 & 1914 \\
\hline 4.300 & 17.250 & 29.100 & 46.300 \\
\hline
\end{tabular}

Les Juifs représentent au début de la guerre $0,6 \%$ de la population belge. D'Ostende à Arlon, l'ensemble des dix communautés juives subit

d'Angélique Burnotte sur le personnage du grand rabbin Bloch durant la Première Guerre mondiale et son sermon de 1916, dans Les Cahiers de la Mémoire contemporaine, 2014, $\mathrm{n}^{\circ} 11$, p. 13- 42.

3 Voir Sophie De Schaepdrijver, «L'Occupation», dans Expo 14-18 c'est notre histoire! Bruxelles, Tempora, 2014, p. 59.

${ }^{4}$ Krzysztof Pomian, « Avant-propos », dans Expo 14-18 ..., ibid., p. 12.

${ }^{5}$ Jean-Philippe Schreiber, L'immigration juive en Belgique du Moyen Ầge à la Première Guerre mondiale, Bruxelles, éditions ULB, 1996, p. 205-210. 
l'occupation allemande. La synagogue principale d'Anvers, inaugurée en 1898, est touchée par un bombardement ${ }^{6}$ survenu le 7 octobre 1914.

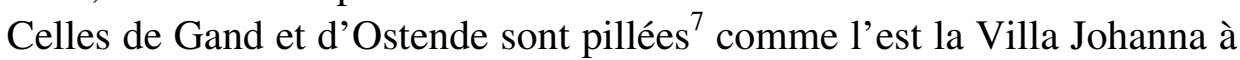
Middelkerke ${ }^{8}$; sans parler des particuliers qui voient l'œuvre de toute une vie détruite par la violence des combats comme, par exemple, l'Alsacien Raphaël Guggenheim qui fait construire à Charleroi, en 1880, le grand magasin éponyme, complètement détruit en 1914.

Parmi le million et demi de Belges qui fuient les combats, dont neuf cent mille rentreront dans leur foyer avant la fin de l'année ${ }^{9}$, on compte douze mille Juifs anversois réfugiés à Londre ${ }^{10}$ - pour la plupart indigents $^{11}$ - et autant en Hollande ${ }^{12}$, répartis entre Scheveningen, Amsterdam et La Haye. Si ceux-ci restent à l'étranger, c'est qu'en tant que ressortissants des puissances ennemies ils ne souhaitent pas prendre les armes contre leur patrie d'accueil ou que leur ressentiment à l'égard de l'Empire russe est trop important.

Quant à l'armée de Belgique, elle n'accepte dans ses rangs que les citoyens belges. Ce n'est qu'à partir de 1915 que l'on fait appel à des volontaires belges ou étrangers. Au total, selon les dernières recherches ${ }^{13}$, on dénombre 280 militaires juifs qui font leur devoir. Du simple soldat au général Louis Bernheim ${ }^{14}$, c'est environ $1 \%$ de la population juive qui est concernée, dont 35 sont tués, soit $12,5 \%$ des engagés.

\footnotetext{
6 Ephraim Schmidt, L'histoire des Juifs à Anvers (Antwerpen), Anvers, édition Excelsior, 1969, p. 123.

${ }^{7}$ Voir le dossier « Dommages de guerre 14-18 », dans A-CCIB.

8 Daniel Dratwa, «De Villa Johanna te Middelkerke (1901-1964)», Heemkring Braningate, vol. 43, ${ }^{\circ} 10$, septembre 1991, p. 98-99.

${ }^{9}$ Voir Sophie De Schaepdrijver, op.cit., p. 57.

10 Ari Joshua Sherman et Pamela Shatzkes, « Otto M. Schiff (1875-1952), Unsung Rescuer », Leo Baeck Institue Year Book, vol.54, 2009, p. 247, citent ce nombre dont seulement «trois milles indigents » reviennent à Anvers au printemps 1919 (voir à ce propos CCIB, Rapport à l'Assemblée Générale des membres effectifs de la Communauté de Bruxelles du 18 avril 1920, Bruxelles, Maison Vve Michel Van Dantzig, 1920, p. 20-21). Pour une vision romanesque de cet exode : Esther Kreitman, Le Diamantaire, Paris, Calman-Levy, 2014, p. 272- 318.

${ }^{11}$ Le rabbin londonien Hermann Gollancz le 24 octobre 1914 et le révérend Herbert Hensley Hanson, le $1^{\text {er }}$ janvier 1915, appelèrent dans leur sermon à s'occuper d'eux comme on peut le lire sur le site http://religionandwar.org/index.htm\#

12 Ludy Giebels, De Zionistische beweging in Nederland 1899-1941, Assen, Van Gorcum \& Comp., 1975, p. 107.

${ }^{13}$ Daniel Dratwa, Les militaires juifs dans l'armée belge en 1914-1918, étude non publiée.

${ }^{14}$ Jean-Philippe Schreiber (sous la dir.de), Dictionnaire biographique des Juifs de Belgique, Bruxelles, de Boeck, 2002.
} 
Dès les premiers jours des hostilités, des rabbins se mettent à la disposition des autorités pour secourir leurs coreligionnaires au sein des services de santé ${ }^{15}$. Armand Bloch, de nationalité française comme Isaac Schwartz et Élie Friedlander, de nationalité anglaise, entrent en fonction le 11 août en vertu de l'arrêté royal du 7 août 1914. Néanmoins, on ne trouve aucun représentant du culte israélite ${ }^{16}$ dans l'ordre de bataille de l'aumônerie du $1^{\mathrm{er}}$ octobre 1914.

Face à l'occupation brutale du pays, de nombreux Juifs de Belgique réagissent. Outre ceux qui rejoignent l'armée pour s'engager au fil du temps, on peut citer quelques actions exemplaires, parmi lesquelles celles de Dannie Heineman ${ }^{17}$ qui aide à la création du Conseil national de secours et d'alimentation pour prévenir une crise alimentaire majeure. Paul Errera, bourgmestre de la commune d'Uccle, améliore le sort de ses administrés malgré les conditions financières précaires; son épouse Isabelle Goldschmidt-Franchetti ${ }^{18}$, l'industriel Alfred Goldschmidt, le banquier Franz Philippson et le médailleur Charles Samuel, pour n'en citer que quatre, jouent un rôle non négligeable en pratiquant une résistance tant effective que spirituelle.

D'autres, une infime minorité, collaborent avec l'ennemi, pour des motifs divers et variés, voire des raisons linguistiques ${ }^{19}$ comme Herman Vos, Salomon Kok, Moïshe Friedman ${ }^{20}$ et le plus célèbre activiste d'entre eux Marten Rudelsheim²1, décédé en prison suite à sa condamnation après la guerre. En effet, membre du Vlaamsch Verbond [Alliance flamande] à Anvers dès 1914, il dirige l'Université populaire de l'Algemeen Nederlandsch Verbond [Association générale néerlandaise] ce qui l'entraîne à siéger pendant dix mois dans le premier Raad van Vlaanderen [Conseil des Flandres] créé par les Allemands jusqu'à la proclamation indépendantiste fin 1917.

\footnotetext{
15 Jacques-Robert Leconte, L'aumônerie militaire belge. Son évolution de l'époque hollandaise à l'organisation actuelle, Bruxelles, Musée Royal de l'armée et d'histoire militaire, 1966, p. 88.

${ }^{16}$ Il n'y a pas d'aumônerie israélite jusqu'en 1928 au sein de l'armée belge malgré les multiples efforts déployés par le Consistoire au fil des ans.

${ }^{17}$ Pour les biographies complètes de toutes les personnes citées dans ce paragraphe voir Jean-Philippe Schreiber, Dictionnaire biographique..., op.cit.

${ }^{18}$ Voir aussi Anne Cherton, «Fonds Errera », dans Musée juif de Belgique - Joods Museum van Belgie, vol. 25, $\mathrm{n}^{\circ}$ 2, p. 6-7 sur les raisons de son incarcération à la prison de Saint-Gilles en 1917.

19 Voir Lieven Saerens, Vreemdelingen in een wereldstad: een geschiedenis van Antwerpen en zijn joodse bevolking (1880-1944), Tielt, Lannoo, 2000, p. 88- 90.

${ }^{20}$ Idem, p. 118

${ }^{21}$ Jean-Philippe Schreiber, Dictionnaire biographique..., op.cit., p. 298-299.
} 
Pour des raisons politiques, on peut citer cet avocat du barreau de Bruxelles, Fritz Norden, qui publie en 1915, La Belgique neutre et l'Allemagne : un livre qui fait grand bruit à l'époque et suscite un certain antisémitisme comme on peut le lire dans La Libre Belgique ${ }^{22}$. En opposition avec la doctrine majoritaire de l'establishment belge, Norden tente d'expliquer que la neutralité proclamée n'a pas de base légale. Cette «démonstration» est jugée tellement satisfaisante par les autorités d'occupation qu'elles l'imposent à la vente dans de nombreuses librairies. Sous la plume de Fidelis (pseudonyme d'Albert van de Kerchove), le journaliste résistant de La Libre Belgique s'en prend à Norden en le traitant de «Juif d'Outre-Rhin » et de «[...] mouchard et hypocrite, c'est dans leur sang [...]»; il conclut son article par ses mots : «Ayant de qui tenir: Judas de la Belgique », qui en disent long sur la mentalité du personnage. Certains continuent à utiliser l'allemand, pour se faire comprendre de la population immigrée germanophone. Si cette pratique est assez courante avant-guerre, durant l'occupation on la perçoit comme un véritable acte de collaboration. On accuse aussi bien le rabbin de Liège, Henri Lehmann, que le rabbin d'Anvers, le Dr Joseph Wiener, d'avoir prêché dans cette langue. D'autres, comme la communauté orthodoxe d'Anvers ou les sionistes bruxellois l'utilisent dans leur publication, comme Hatikvah.

La coopération avec l'ennemi permet aussi à certains de bénéficier d'avantages abusifs. Ce qui est perçu comme un comportement révoltant puisqu'il s'agit parfois de responsables du culte comme le rabbin de Liège $^{23}$ - déjà cité - qui est «blanchi » ${ }^{24}$, et de Moses Goldstein de la communauté israélite orthodoxe de Bruxelles qui « sera démissionné » en 1918.

$\mathrm{Au} \mathrm{vu}$ des faits reprochés, ces réactions peuvent paraître disproportionnées, mais il faut se rappeler que le patriotisme exacerbé et

\footnotetext{
${ }^{22}$ Je tiens à remercier vivement Yasmina Zian qui a attiré mon attention sur cet article que l'on peut lire in extenso sur http://bildungsblog.hypotheses.org/97.

${ }^{23}$ Lettre du $1^{\text {er }}$ mars 1919 du secrétaire général du ministère de la Justice au président du Consistoire (A-CCIB, boîte ministère de la Justice 1910-1932, dossier « Divers avril 1910-mars $1920 »)$ l'accusant d'avoir entre autres : «rencontré à Aix-la-Chapelle le rabbin Heinrich Jaulius pour recevoir ses ordres, d'en être revenu avec un brassard allemand puis d'avoir trafiqué au marché noir des pommes de terre et la graisse d'animaux rituellement abattus $[\ldots]$ ». Malgré cela, il reste en poste jusqu'à son décès en 1933.

${ }^{24}$ Après ses déclarations et des preuves documentées, il est absout des charges qui pèsent sur lui par le CCIB lors de sa séance du 11 février 1919: voir Registre des Procès-Verbaux $\mathrm{n}^{\circ} 8$, p. 1-4 dans A-CCIB.
} 
la grande pauvreté affectant une importante partie de la population juive $^{25}$ et non juive font que le moindre faux-pas est sévèrement jugé par l'opinion publique.

Les batailles des premiers mois de la guerre frappent profondément les familles. Celles-ci, endeuillées ou affectées par les blessures d'un proche, ont en outre à faire face à la disette et au ralentissement général de l'activité économique. Toutes ces difficultés, cette douleur, et ces malversations arrivent aux oreilles du grand rabbin Armand Bloch qui est lui-même durement touché par les conditions si particulières de la mort du grand rabbin de Lyon Abraham Bloch, le 29 août 1914. Ce frère aîné et aimé était aumônier militaire sur le front français, malgré son âge. Il est tué en apportant un crucifix à un soldat gravement blessé ${ }^{26}$, selon la légende ${ }^{27}$.

Malgré la dureté des temps, quelques pièces d'archives ont laissé la trace d'une continuité de la vie religieuse et sociale, sans empêcher la diversité des tendances. Ainsi en est-il de la fête de Pourim ${ }^{28}$ célébrée le 19 mars 1916 par la Jeunesse israélite de Bruxelles suivie d'une tombola au profit de la Société israélite de Bienfaisance ou de la fête de Hanoucca 5678 organisée pour les familles par les Naare-Sion, proche de la communauté principale, le 6 novembre 1917 au café du Cygne ${ }^{29}$ alors que le Comité central sioniste de Bruxelles, proche de la communauté orthodoxe, organise «sa » fête le dimanche 16 décembre 1917 (avantdernier jour de Hanoucca) à l'hôtel de Bordeaux ${ }^{30}$.

Ce climat et ces faits permettent de mieux apprécier ce qui a poussé une personnalité, par nature réservée, à se mettre en danger et à devenir «l'âme de la résistance morale » ${ }^{31}$. Mais qui est donc le grand rabbin Armand Bloch et quelle est son action ? Telles sont les questions que l'on

\footnotetext{
${ }^{25}$ La population des assistés de la Société Israélite de Bienfaisance de Bruxelles passe de 339 personnes en août 1914 à 766 en décembre 1914. Voir le Rapport du conseil d'administration pour l'année 1914. Actions de la Société pendant la guerre, Bruxelles, Imprimerie Maison Vve Michel Van Dantzig, 1915, p. 8.

${ }^{26}$ Paul Netter, Un grand rabbin dans la Grande Guerre, Triel-sur-Seine, Italiques, 2013, p.100-102.

${ }^{27}$ Pierre Birnbaum, «French Rabbis and the 'Sacred Unity' during the First World War», European Judaism, vol. 48, n 1, 2015, p. 51.

${ }^{28}$ Archives du MJB (AMJB) numéros 06504 et 03722.

${ }^{29}$ AMJB n ${ }^{\circ} 08961$.

${ }^{30}$ AMJB n ${ }^{\circ} 08962-08963$.

${ }^{31}$ Willy Bok, «Vie juive et communauté. Une esquisse de leur histoire au vingtième siècle » dans La Grande synagogue de Bruxelles 1878-1978, Bruxelles, Communauté israélite de Bruxelles, 1978, p. 154.
} 
est en droit de se poser quand on connaît l'importance de cette fonction à la fin du XIX ${ }^{\mathrm{e}}$ siècle $^{32}$.

Né à Saint-Mandé le 17 juin 1861, Armand Bloch fit de solides études au séminaire israélite de Paris. Après sa sortie de l'école rabbinique en 1884, il exerça comme bibliothécaire à l'Alliance israélite universelle (AIU) pendant deux ans avant de se consacrer, durant cinq ans, au ministère rabbinique de Toul (Meurthe-et-Moselle). C'est durant sa vie parisienne qu'il rencontra celle qui devint sa femme : la petite-fille du Dr. Henri Loeb, ancien grand rabbin de Belgique et dont la fille avait épousé le compositeur Jules Erlanger, cofondateur de l'Alliance israélite universelle, administrateur de la communauté israélite de Bruxelles et auteur de son répertoire liturgique. On comprend mieux, dans ces conditions, pourquoi la candidature d'Armand Bloch fut si facilement acceptée.

À peine âgé de trente ans, le rabbin se donna immédiatement de toutes ses forces à sa nouvelle tâche qui était vaste car le développement des communautés et l'accroissement de la population juive avaient créé des charges supplémentaires. Le grand rabbin donna une réponse adéquate grâce à la collaboration précieuse de philanthropes généreux et dévoués. Durant son mandat, cinq synagogues furent construites et décorées : trois à Anvers, une à Liège et une à Ostende. Comme membre important de l'AIU, il fut le moteur des protestations contre les persécuteurs des Juifs dans divers pays comme, par exemple, la Russie ${ }^{33}$ ou la France. Pour ce pays, peu après l'affaire Dreyfus, il conclut, le 13 décembre 1903, à Hanoucca, une conférence intitulée: «L'esprit militaire des Juifs », après avoir cité de nombreux exemples historiques, que les Juifs « considèrent comme le premier des devoirs, avant même le devoir religieux, le devoir envers la patrie ». Il faut cependant remarquer que, comme d'autres rabbins européens confrontés à l'éveil du sentiment nationaliste présent au sein du peuple juif, son opposition au sionisme ne fit que s'affirmer. Aussi les sionistes belges, et principalement ceux d'Anvers, répondirent-ils souvent avec peu d'aménité, dans leurs journaux, aux déclarations du chef religieux du judaïsme belge, avant de contrer peu à peu son courant en prenant le contrôle des communautés ${ }^{34}$.

\footnotetext{
${ }^{32}$ Les renseignements suivants proviennent de l'article de l'auteur, «Armand Bloch, grand rabbin de Belgique », Tsafon, n 18, 1994, p. 23-33.

${ }_{33}$ Voir son sermon intitulé Les Juifs russes prononcé le 9 décembre 1893, Bruxelles, Imprimerie Vve Michel Van Dantzig, 1893, 15 p.

34 Jean-Philippe Schreiber, Politique et religion. Le Consistoire Central israélite de Belgique au XIX ${ }^{e}$ siècle, Bruxelles, éd. de l'université de Bruxelles, 1995, p. 364-367.
} 
Président ou membre de droit de tous les comités, organisations ou œuvres charitables de la communauté israélite de Bruxelles, Armand Bloch déploya son zèle et sa sollicitude dans toutes ses activités. On se doit de souligner son attention particulière et ses efforts dans la lutte contre la tuberculose par la création de la Villa Johanna et de la Société israélite d'Assistance antituberculeuse. Afin de trouver plus de fonds pour des œuvres sociales, il innova en faisant créer par l'artiste Constant Montald un timbre en 1910.

Nonobstant ses lourdes charges au profit de la charité et de la philanthropie qui l'entraînaient souvent à travailler jusqu'à une heure avancée de la nuit, il n'oubliait aucunement les devoirs liés à sa fonction. Malgré la très grande qualité de ses sermons, il devait user sans cesse de ménagements et d'habiles interventions pour ramener aux offices synagogaux ses fidèles en voie de déjudaïsation. Comme la plupart des autres pays européens, la Belgique ne fit pas exception à ce phénomène qui est lié à l'attitude libérale envers les Juifs qu'adoptèrent de nombreux gouvernants depuis la Révolution française. Face à cette déception, il reporta ses espoirs sur la jeunesse. II devint, grâce à son travail opiniâtre, un maître avisé prenant joie à prodiguer son enseignement religieux et moral. Il préparait avec attention ses élèves, garçons et filles, pour la cérémonie de l'initiation ${ }^{35}$ dont il escomptait les meilleurs effets pour la foi et le judaïsme. On lui doit d'ailleurs un manuel qui fut en usage jusqu'a la fin de la Seconde Guerre mondiale ${ }^{36}$.

Ses multiples activités dans tant de domaines le firent promptement reconnaître en dehors des cercles juifs. Dès le 24 novembre 1892, il est appelé à la vice-présidence du conseil des veuves et orphelins au ministère de la Justice. Le 31 décembre 1905, il est nommé inspecteur de la religion et de la morale dans les écoles primaires. Les honneurs s'ajoutèrent bientôt à ces distinctions : il est fait chevalier de l'Ordre de Léopold le 10 mai 1905 et promu, le 15 décembre 1919, officier avec rayure d'or, en récompense de sa «valeureuse conduite civique et des services rendus, à l'heure douloureuse de l'occupation de la patrie par l'ennemi ».

En effet, au mois d'octobre 1914, le gouverneur général allemand adresse à tous les fonctionnaires, y compris le clergé, des contrées

\footnotetext{
35 Daniel Dratwa, « $\mathrm{A}$ propos des certificats d'initiation religieuse : analyse iconographique et socio-culturelle de 1842 à nos jours », MuséOn, n 3, 2011, p. 56-58.

${ }^{36}$ Armand Bloch, Éléments d'instruction religieuse et morale à l'usage de la jeunesse israélite, Paris, Librairie Durlacher, 1912.
} 
occupées l'ordre de signer, conformément aux conventions internationales de La Haye, une déclaration par laquelle le fonctionnaire s'engage à ne rien commettre et à tout omettre qui puisse être préjudiciable à l'autorité occupante ${ }^{37}$.

Armand Bloch, en qualité de chef du culte israélite, est chargé de réunir les engagements de tous les rabbins et ministres officiants placés sous son autorité. Comme le clergé catholique, après diverses interventions - suite aux incidents auxquels donne lieu la lecture de la lettre pastorale du cardinal Mercier - est dispensé de signer l'engagement demandé, le grand rabbin de Belgique refuse également jugeant qu'il n'est pas acceptable de placer le culte israélite dans une situation d'infériorité car la Constitution belge met tous les cultes sur un pied d'égalité, ainsi que l'établit le professeur Paul Errera, éminent jurisconsulte $^{38}$. Le gouverneur général allègue que la situation des deux cultes n'est pas assimilable et continue de suspendre les traitements des fonctionnaires du culte israélite bruxellois ${ }^{39}$. Dans les provinces, leurs collègues signent l'ordre à la suite d'un malentendu.

En dépit de l'attitude de l'autorité occupante, lors de la cérémonie de Yom Kippour en 1915 en présence du rabbin de Darmstadt et de dix soldats, le grand rabbin Bloch délivre un sermon patriotique (qui a été perdu) avant de prononcer la prière pour le roi Albert et de faire jouer la Brabançonne $^{40}$.

Malgré de nombreuses pressions et un entretien ${ }^{41}$, le 19 octobre 1915, avec M. von Friedberg, représentant l'autorité provinciale du Brabant, le guide spirituel du judaïsme belge campe sur ses positions en

37 Copie de la lettre du 5 décembre 1918 du président du Consistoire à Émile Vandervelde, ministre de la Justice, A-CCIB, ${ }^{\circ} 169$ du Registre des Correspondances.

${ }^{38}$ Voir le rapport de trois pages tapées à la machine avec le titre manuscrit: Note sur la situation légale $d u$ culte en Belgique, dans A-CCIB dossier «lettres diverses 1950$1955 »$.

${ }^{39}$ Ils reçoivent leur salaire, dû depuis juillet 1914, seulement en mai 1919 ; voir à ce propos la lettre du 12 avril 1919 d'Émile Vandervelde, ministre de la Justice au Consistoire, A-CCIB dossier « lettres diverses 1950-1955».

${ }^{40}$ Cette information provient d'une coupure de presse retrouvée dans les archives du rabbin David Berman, gendre du rabbin Bloch, déposées par celui-ci au Musée Juif de Belgique. Quant au «rabbin de Darmstadt », il s'agit très probablement de l'aumônier Bruno Italiener (1881-1956) comme me l'a confirmé Peter Appelbaum que je remercie ici ; cf. son livre Peter Appelbaum, Loyalty Betrayed : Jewish Chaplains in the German Army During the First World War, Londres, Valentine Mitchell, 2014, p. 121-144.

${ }^{41}$ Rapport manuscrit de trois pages du grand rabbin dans lequel il consigne son entretien et le date à deux reprises du 19 octobre 1915, contrairement à la lettre du 5 décembre 1918 dans laquelle il est noté, par le président du Consistoire, que celui-ci a eu lieu en février 1916 ; voir A-CCIB dossier « Lettres diverses 1950-1955 ». 
refusant le moindre écart au principe de l'égalité constitutionnelle des cultes. Dès lors, il est tenu pour suspect car, outre son refus d'obéissance, il continue à réciter la prière pour le roi lors des services du culte, suivie de l'exécution de la Brabançonne. Cette attitude patriotique mécontente un grand nombre de soldats israélites allemands.

Aussi quand, le 18 avril 1916 premier jour de la Pâque, Armand Bloch développe le texte rituel sur l'esclavage et la liberté en l'intitulant « les idoles modernes », publié à Paris, en $1920^{42}$, la limite est atteinte. En effet, dans l'esprit de cette fête, le grand rabbin choisit de développer le sujet du Décalogue, car celui-ci est :

Le premier de tous les commandements, celui qui, par conséquent, doit revêtir le caractère le plus important, [qui] nous ordonne de croire en Dieu [...] qui a brisé les chaînes de la servitude d'Égypte. ${ }^{43}$

Il poursuit en soulignant que ce n'est pas «la Loi des seuls Israélites » mais c'est « la charte de l'Humanité » car :

C'est l'indépendance que la sortie d'Égypte, que le Décalogue a apportée au monde, la liberté de penser et de croire, le droit des consciences, le droit de sentir et d'aimer selon son cœur, le droit de juger suivant sa raison. Nous sommes des êtres libres et nous avons le devoir de rester libres, de défendre, de sauvegarder notre indépendance, de ne pas la sacrifier honteusement, pour des considérations indignes de nous. ${ }^{44}$

Il faut renverser la force, l'idole moderne par excellence, assène-til, car elle nous fait devenir :

Esclaves de la crainte ou de l'intérêt, esclaves par calcul, par bassesse ou lâcheté, voilà les espèces qui se rencontrent, hélas ! Si fréquemment, au milieu de nous. ${ }^{45}$

Et il continue par ces mots :

\footnotetext{
${ }^{42}$ Armand Bloch, Les Idoles Modernes. Sermon prononcé à la synagogue de Bruxelles le $1^{\text {er }}$ jour de Pâque 5676, 18 avril 1916, Paris, Librairie Durlacher, 1920. De nos jours, on peut aussi lire ce texte dans Les Cahiers de la Mémoire contemporaine, op.cit., p. 35- 42 ou sur http://religionandwar.org/index.htm\# ou sur http://www.rachelnet.net/media/aiu/livres/FR_AIU_L_8JBR679.pdf .

${ }^{43}$ A. Bloch, Ibid., p. 8.

${ }^{44}$ Ibid., p. 11.

${ }^{45}$ Ibid., p. 11.
} 
Honneur à ceux qui triomphent par la justice et honte à ceux qui applaudissent au succès déloyal, honte à ceux qui flattent les puissants, alors qu'ils ont foulé aux pieds la justice et le droit. Ce ne sont pas des hommes libres. Ce sont des esclaves. ${ }^{46}$

En fin de sermon, il établit le lien avec le présent :

Nous traversons des heures semblables. L'indépendance, jamais nous ne l'avons aussi bien comprise, jamais autant appréciée, autant aimée, et notre plus grand bonheur serait de la voir, demain, universellement reconnue et respectée, comme le droit le plus sacré. ${ }^{47}$

Poursuivant son insoumission, il dénonce toutes les bassesses, dont il a eu connaissance depuis le début de l'occupation, et il invite ses ouailles, en des termes dénués d'ambiguïté, à rejoindre les rangs de ceux qui luttent déjà :

Cette volonté d'indépendance, il faut qu'elle devienne universelle. La sortie d'Égypte n'aura accompli tous ses effets que lorsque les chaînes de l'esclavage auront été brisées partout. ${ }^{48}$

Finalement, comme les prophètes bibliques, il proclame, se référant à la tradition :

Aux premières lignes de l'Haggadah que nous lisions hier soir, au seder familial, nous disions: «cette année-ci nous sommes esclaves, l'année prochaine nous serons libres». Oui! L'année prochaine nous serons libres! Nous aurons souffert longuement, mais nous aurons appris à aimer les belles causes, à endurer pour elles les privations, les dangers, les douleurs. Nous serons libres, parce que nous aurons mérité notre liberté ! Amen $!^{49}$

Huit ans plus tôt, en 1908 au même endroit et pour la même occasion, le grand rabbin avait déclamé un texte ${ }^{50}$ très proche, excepté pour les conclusions, et l'avait clôturé en exhortant la jeunesse à être fidèle à la tradition de ses aïeux. Mais les temps ont changé !

\footnotetext{
${ }^{46}$ Ibid., p. 13.

${ }^{47}$ Ibid., p. 14.

${ }^{48}$ Ibid., p. 16.

${ }^{49}$ Ibid., p. 16.

${ }^{50}$ Armand Bloch, Indépendance. Sermon prononcé au temple israélite de Bruxelles, le $1^{\text {er }}$ jour de Pâque 5668 (16 avril 1908), Imprimerie-papeterie Vve Michel Van Dantzig, Bruxelles, 1908, $18 \mathrm{p}$.
} 
II est dénoncé pour outrage par Hugo Wurzburger, attaché à la police secrète allemande, et Fritz Roos, sous-officier dans la Deutsches Heer. Ce qui est normal après tout car ils sont des patriotes allemands avant d'être Juifs comme Armand Bloch l'est dans l'autre camp. Le conseil de guerre devant lequel il est déféré le 10 mai 1916 lui inflige une peine de près de six mois d'emprisonnement «für beleidigung », pour insulte, jusqu'au $1^{\mathrm{er}}$ octobre $^{51}$. Malgré la lettre du Consistoire au général gouverneur baron von Bissing du 15 mai 1916 de ne pas exécuter la sentence et l'intervention du grand rabbin de Cologne M. Wolff, le grand rabbin de Belgique est écroué, dès le lendemain, à la prison de SaintGilles. Son stoïcisme et sa bonne humeur lui permettent de se lier d'amitié avec certains de ses compagnons de captivité comme le R. P. jésuite Devroye du collège Saint-Michel et le chanoine Cocheteux de Saint-Jacques-sur-Coudenberg ${ }^{52}$.

Quelques jours plus tard, il devient une sorte de « héros » aux yeux de la presse $^{53}$ résistante même si le 4 juin on l'autorise à sortir pour célébrer le mariage de Henri Nort et Charlotte Huisman. Il ne peut sortir pour célébrer la fête de Shavouot mais il est présent le 16 juillet à la synagogue pour superviser l'initiation religieuse de ses élèves.

Armand Bloch subit son emprisonnement jusqu'au 28 septembre, date à laquelle il est autorisé à reprendre son service à cause des grandes solennités juives et parce qu'il n'y a aucun autre ministre du culte comme le Consistoire l'a souligné dans ses nombreuses lettres. Il semble qu'aucun militaire juif allemand n'assiste au service de Roch Hachana conduit par le grand rabbin parce que leurs aumôniers ont réquisitionné le théâtre municipal de Saint-Quentin (Aisne) devant lequel 1.600 d'entre eux se font photographier ${ }^{54}$.

\footnotetext{
${ }^{51}$ Voir la lettre du 22 août 1916 du Gericht des General-Gouvernements in Belgien au CCIB dans A-CCIB, Registre des Correspondances, $\mathrm{n}^{\circ}$ 73. Je souhaite remercier la secrétaire du CCIB, Danielle Sansoglou, pour son intérêt pour mes recherches.

${ }^{52}$ M. G. (auteur non identifié), « Armand Bloch, 1861-1923 », Kehilatenou, n 31, juin 1951, p. 3.

${ }^{53}$ Pour une revue détaillée de la presse résistante à ce sujet, voir Angélique Burnotte, «Armand Bloch, le rabbin Patriote », Les Cahiers de la Mémoire contemporaine, $\mathrm{n}^{\circ} 11$, 2014 , p. 28-31. Je remercie vivement l'auteur d'avoir bien voulu partager le résultat de sa recherche.

${ }^{54}$ Lors de la conférence à Londres mentionnée plus haut, ma visite au Jewish Museum me permit de découvrir une photo montrant plusieurs centaines de soldats juifs devant un théâtre, datée de 1916 et localisée à Bruxelles. À la suite de recherches avec mon collègue Philippe Pierret, que je remercie, il est probable que la photo a été prise autour du 28 septembre. Elle fut publiée la première fois dans Allgemeine Zeitung des Judentums du 10 novembre 1916, p. 535-536, comme illustration de l'article de
} 
Après la fin de la tourmente ${ }^{55}$ et les secousses profondes qu'elle a engendrées (en Allemagne, en Autriche, la Révolution russe et ses conséquences...) les efforts du guide spirituel du judaïsme belge se déploient dans l'œuvre de reconstruction (remplacement de fonctionnaires du culte, œuvres sociales, dossiers de dommages de guerre, séquestres ${ }^{56} .$. ) et d'aide aux nombreux migrants d'Europe orientale et d'Allemagne qui arrivent en Belgique et s'y installent depuis que les USA leur ont fermé les portes.

Mais son emprisonnement, sans doute, et le surcroît de travail minent sa santé ; après une longue maladie, la mort le frappe le 30 août 1923. Les honneurs militaires sont rendus au défunt lors de ses obsèques solennelles le 3 septembre. On peut encore voir sa sépulture au cimetière israélite de Kraainem.

l'aumônier militaire Martin Salomonski. La photo de Londres est donc une copie de la photo publiée dans le livre de Martin Salomonski, Ein Jahr an der Somme, Francfortsur-l'Oder, Trowitzsch und Sohn, 1917, planche $n^{\circ} 6$. Je remercie chaleureusement Peter Appelbaum pour cette référence ; il reproduit ce cliché, planche 26, dans son livre déjà cité.

55 Les soldats juifs allemands, prisonniers de guerre à Anvers, sont autorisés, grâce à l'intervention du grand rabbin, à assister aux services de la synagogue principale de cette ville; voir CCIB, Rapport à l'assemblée générale des membres effectifs de la Communauté de Bruxelles du 18 avril 1920, Bruxelles, Vve Michel Van Dantzig, 1920, p. 8 .

56 Parmi tous ceux-ci citons les séquestres des magasins Leonhard Tietz à Anvers et Bruxelles, celui d'Edouard Balser et des Juifs turcs d'Anvers. 
Les Juifs belges de Londres remercient leur bienfaiteur Otto Schiff

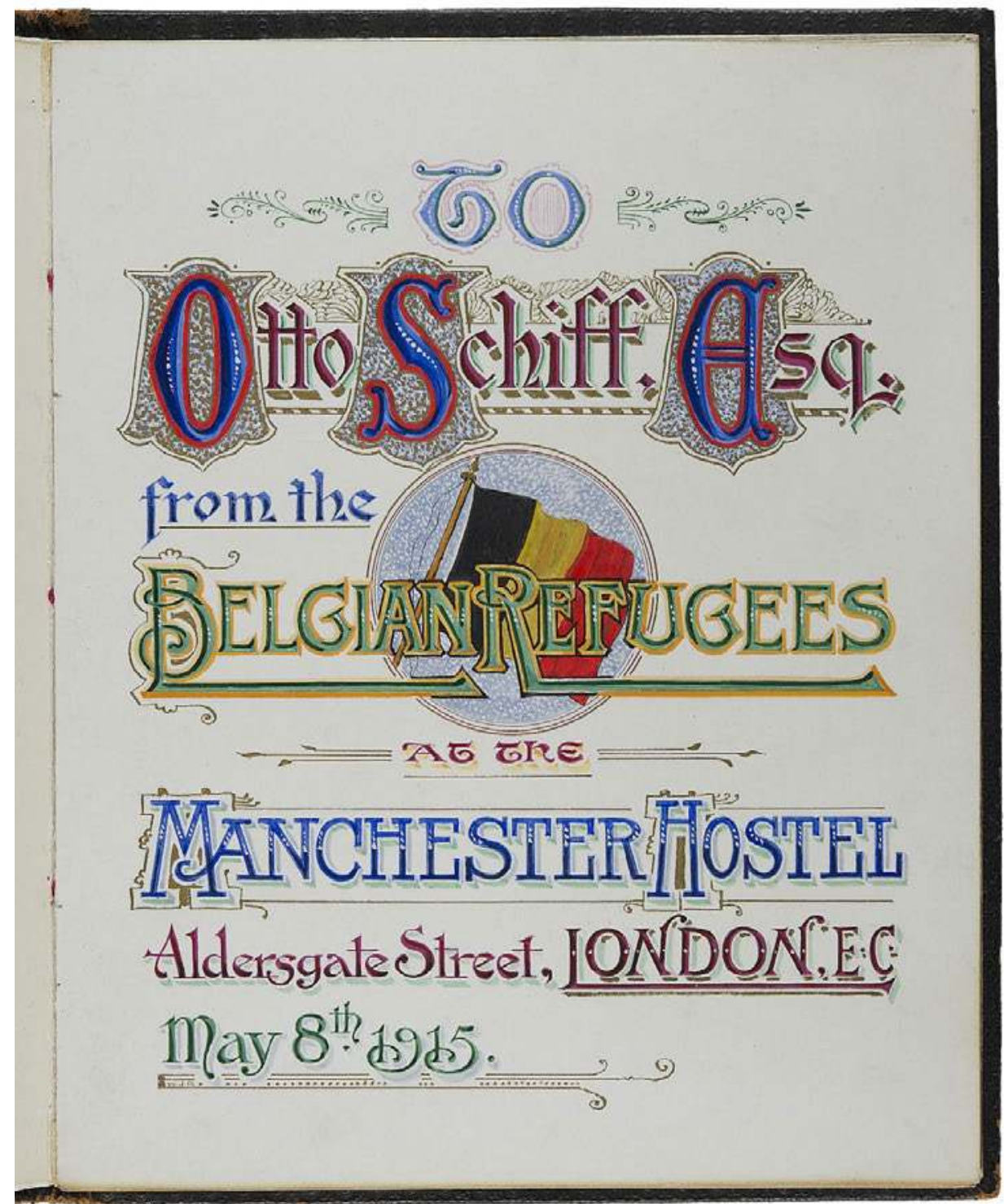

Avec l'aimable autorisation d'Élizabeth Selby du Jewish Museum de Londres Inv. $\mathrm{N}^{\circ} 2003.24$. 
Des soldats allemands dans la salle consistoriale de la synagogue de Bruxelles

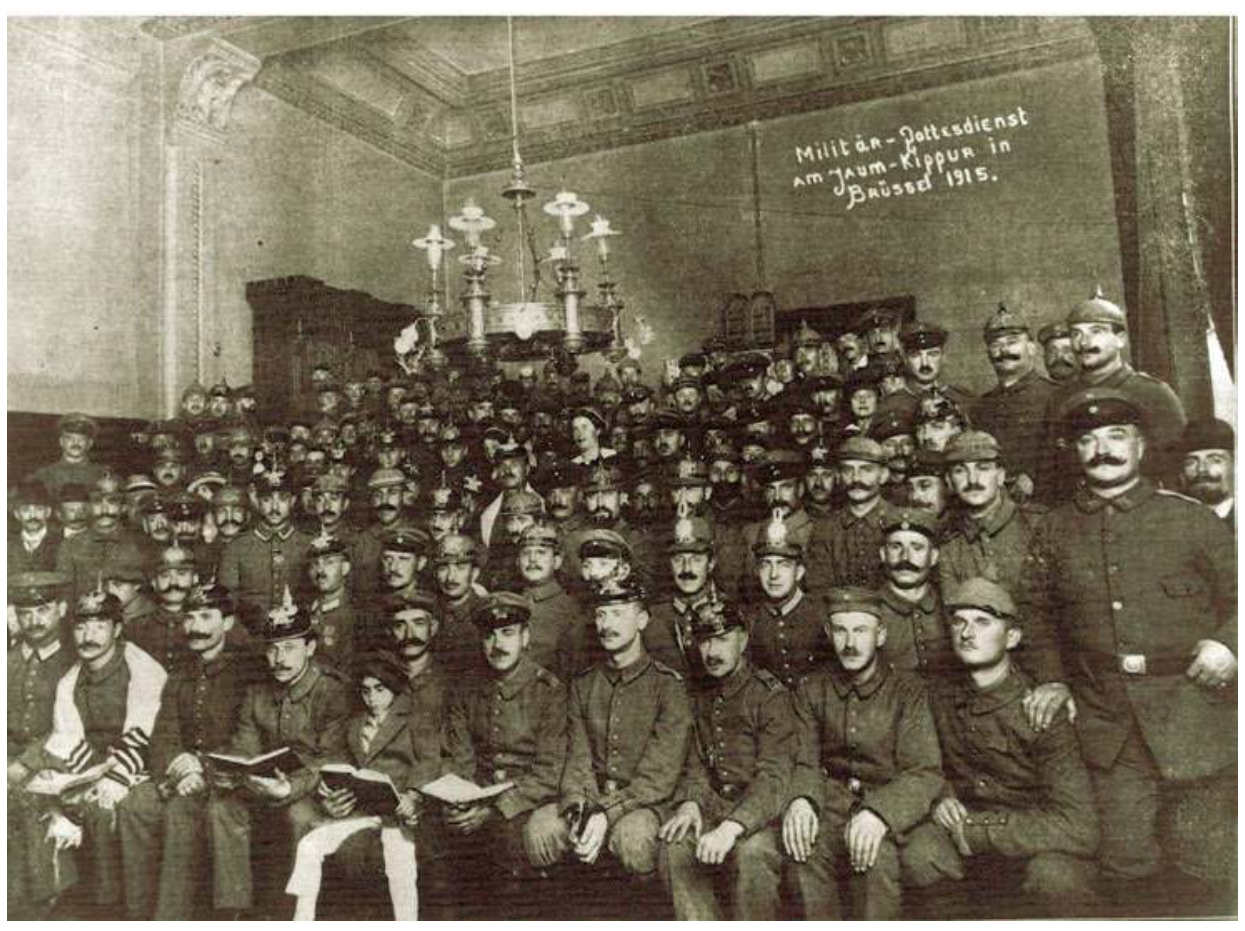

Avec l'aimable autorisation du Leo Baeck Institute de New York 
Mémorial aux soldats juifs

\section{Communauté ISRAÉLITE dE BRUXELLES}

1914

AH Paul

BEN SADOUN Haïm

BEROLZHEIMER René

BLOCH Jules

Caen-Laueunesse Georoges

DEUTSCH Pierre

ECKSTEIN Serğe

Frank Emmanuel

GOLDSTEIN Fernand

GRYNROCK Simon

HARTOG Philippe

LEMER Arthur

LEVY-SCHWAB Gaston

LEVY Howard

LEVY Roudi

Pevtschin Léon

\section{POUR LA PATRIE-}

1918

MAISONPIERRE Jules

MEYER Georges

MEYER Martin

MICHEL Paul

NATAN Robert

OPPENHEIM Alfred

PHILIPPSON Jacques

ROTHSCHILD PaUI

SALOMON Robert

SCHWARTZ Isaac Rabbin

SCHWARTZ Roger

VANDER HAM Harry

VAN PRAaG Joseph

WALEWYK Marcel

ZARMATI Moise

Van Straeten Camille

WURMS Arthur

(C) Daniel Dratwa 